\title{
Um relato de caso sobre exercício físico e Diabetes Mellitus Tipo II: ainda podemos nos surpreender?
}

\section{A Case Report on Exercise and Type II Diabetes Mellitus: Can We Still Be Amazed?}

\author{
Douglas Gibran Lobo do Espírito Santo Cerqueira' ${ }^{1}$ (C) \\ Marvyn de Santana do Sacramento 2 (1) \\ Viviane Rocha dos Santos ${ }^{3}$ (1) \\ Tailma Costa de Jesus ${ }^{4}$ (1) \\ Igor Macedo de Oliveira ${ }^{5}$ \\ Jefferson Petto ${ }^{6}$ (1)
}

'Centro Universitário Social da Bahia (Salvador), ACTUS CORDIOS Reabilitação Cardiovascular, Respiratória e Metabólica (Salvador). Bahia, Brasil. douglas.gibran@hotmail.com ${ }^{2}$ Centro Universitário Social da Bahia (Salvador), ACTUS CORDIOS Reabilitação Cardiovascular, Respiratória e Metabólica (Salvador), Faculdade do Centro Oeste Paulista (Bauru). Bahia, São Paulo, Brasil. marvynsantana@gmail.com ${ }^{3}$ Centro Universitário Social da Bahia (Salvador). Bahia, Brasil. vivirocha92@yahoo.com ${ }^{4}$ Autora para correspondência. Centro Universitário Social da Bahia (Salvador). Bahia, Brasil. taailmacosta@hotmail.com ${ }^{5}$ Faculdade Ruy Barbosa (Salvador), ACTUS CORDIOS Reabilitação Cardiovascular, Respiratória e Metabólica (Salvador). Bahia, Brasil. igormacedoliveira@hotmail.com ${ }^{6}$ Escola Bahiana de Medicina e Saúde Pública (Salvador), Centro Universitário Social da Bahia (Salvador), Faculdade Adventista da Bahia (Cachoeira), Bahia, Brasil. ACTUS CORDIOS Reabilitação Cardiovascular, Respiratória e Metabólica (Salvador). Bahia, Brasil. petto@cardiol.br

RESUMO | INTRODUção: O Diabetes mellitus tipo 2(DM2) é fator de risco independente para o desenvolvimento de doenças cardiovasculares. $\mathrm{O}$ exercício físico é uma terapêutica adjuvante e eficaz no controle do DM2, pois evita o declínio funcional, complicações cardiovasculares e melhora a qualidade de vida. OBJETIVO: Apresentar a repercussão de um programa de reabilitação cardiovascular e metabólica sobre o controle glicêmico e capacidade funcional em uma paciente com DM2 e insuficiência cardíaca classe III. MÉTODOS: Trata-se de um relato de caso envolvendo uma mulher de 63 anos, irregularmente ativa, eutrófica, sarcopênica, hipertensa e com DM2 em uso de insulina (NPH e ultrarrápida) há 6 anos, com diagnóstico de insuficiência cardíaca(IC) classe funcional III. Ingressa em programa de reabilitação cardiovascular e metabólica supervisionado. Realiza avaliação de perfil lipídico, hemoglobina glicada, teste de caminhada de 6 minutos (TC6), glicemia de jejum. Durante a sessão foi monitorizada a glicemia capilar, pressão arterial sistêmica (PAS) e o traçado eletrocardiográfico. O protocolo consistia em alongamento, exercícios neuromusculares e esteira ergométrica realizados com carga de 12-14 da escala de Borg. O programa durou 16 semanas, sendo realizado 2 vezes na semana totalizando 80 min por sessão. RESULTADOS: Houve acréscimo de $128,57 \%$ no TC6 (350 vs $800 \mathrm{~m}$ ). Os valores da hemoglobina glicada pré e pós-programa de treinamento foram $12 \%$ vs-7,5\%; glicemia de jejum 346mg/dL-vs-105mg/dL; colesterol total 158mg/ dL-vs-108mg/dL; lipoproteína de baixa densidade(LDL) 95mg/dL-vs-58mg/ $\mathrm{dL}$; lipoproteína de alta densidade (HDL) $31 \mathrm{mg} / \mathrm{dL}-\mathrm{vs}-41 \mathrm{mg} / \mathrm{dL}$; triglicerídeos $115 \mathrm{mg} / \mathrm{dL}$-vs-97mg/dL e PA $185 \times 95 \mathrm{mmHg}$ vs $139 \times 85 \mathrm{mmHg}$. Ao final do programa foi retirada a utilização da insulina subcutânea. CONCLUSÃo: O programa demonstrou-se adequado na melhora da capacidade funcional submáxima e no controle dos níveis glicêmicos e lipídicos plasmáticos.

PALAVRAS-CHAVE: Síndrome metabólica. Reabilitação cardíaca. Glicemia. Qualidade de vida.
ABSTRACT | INTRODUCTION: Diabetes mellitus type 2 (DM2) is an independent risk factor for the development of cardiovascular diseases. Physical exercise is an adjuvant and effective therapy in the control of DM2, as it prevents functional decline, cardiovascular complications and improves quality of life. OBJECTIVE: To present the repercussions of a cardiovascular and metabolic rehabilitation program on glycemic control and functional capacity in a patient with DM2 and class III heart failure. METHODS: This is a case report that involved a 63-year-old woman, irregularly active, eutrophic, sarcopenic, hypertensive and with DM2 on insulin (NPH and ultrafast) for 6 years, diagnosed with heart failure (HF) functional class III. Joins a supervised cardiovascular and metabolic rehabilitation program. Performs lipid profile, glycated hemoglobin, 6-minute walk test (6MWT), fasting glucose. During the session, capillary blood glucose, blood pressure (BP) and electrocardiographic tracing were monitored. The protocol consisted of stretching, neuromuscular exercises and a treadmill performed with a load of 12-14 on the Borg scale. The program lasted 16 weeks, being carried out twice a week totaling 80 min per session. RESULTS: There was an increase of $128.57 \%$ in the 6 MWT ( 350 vs $800 \mathrm{~m}$ ). The glycated hemoglobin values before and after the training program were $12 \%$-vs-7.5\%; fasting blood glucose 346mg / dL-vs-105mg / dL; total cholesterol 158mg / dL-vs-108mg / dL; lowdensity lipoprotein (LDL) 95mg / dL-vs-58mg / dL; high density lipoprotein (HDL) $31 \mathrm{mg} / \mathrm{dL}-\mathrm{vs}-41 \mathrm{mg} / \mathrm{dL}$; triglycerides 115mg / dL-vs-97mg / dL and PA $185 \times 95 \mathrm{mmHg}$ vs $139 \times 85 \mathrm{mmHg}$. At the end of the program, the use of subcutaneous insulin was withdrawn. CONCLUSION: The program proved to be adequate in improving submaximal functional capacity and in controlling plasma glycemic and lipid levels.

KEYWORDS: Metabolic syndrome. Cardiac rehabilitation. Blood glucose. Quality of life. 


\section{Introdução}

A diabetes mellitus (DM) é a síndrome metabólica com maior prevalência no mundo, com expectativa de projeção para 592 milhões em 2035. No Brasil mais de 60\% das mortes estão associadas a doenças cardiovasculares o que alerta para importância de medidas preventivas visto o grande impacto social e econômico gerado por essas enfermidades, sendo a DM uma de suas principais causas. Além das doenças cardiovasculares a DM é causa de amputações, neuropatias, falências de órgãos e doença renal crônica ${ }^{1,2}$. É descrito também que as doenças cardiovasculares representam a maior causa de morte em pacientes com diabetes melitus tipo 2 (DM2) em função das alterações desfavoráveis da glicemia, insulina e perfil lipídico destes pacientes, bem como da associação com a hipertensão arterial sistêmica (HAS) e inflamação².

Uma das formas de controle dos níveis glicêmicos é através de terapia farmacológica, mas, a forma de aplicação e a frequência representam uma das principais queixas entre os pacientes ${ }^{3}$. O exercício físico, por sua vez, é uma terapêutica adjuvante e eficaz no controle do DM2, pois, além de evitar o declínio funcional, aumenta a sensibilidade do organismo à insulina, previne complicações renais, cardiovasculares, neuromusculares e melhora a qualidade de vida ${ }^{3,4}$.

Apesar do reconhecimento sobre a importância do exercício físico, como demonstrado pela Sociedade Brasileira de Diabetes, pouco se tem relatado sobre o seu efeito potencial na remoção de terapias farmacológicas com um programa estruturado e individualizado de reabilitação cardiometabólica ${ }^{4,5}$. Portanto, este relato de caso apresenta a repercussão de um programa de reabilitação cardiovascular e metabólica supervisionada sobre o controle glicêmico e capacidade funcional de uma paciente com DM2 e insuficiência cardíaca classe III.

\section{Descrição do caso}

O presente relato de caso foi avaliado pelo Comitê de Ética e Pesquisa da Universidade Estadual de Feira de Santana sob o número 033/2011 (CAAE 0036.059.00011) conforme CNS 196/96, vigente à época.

\section{Dados do paciente}

CLES, 65 anos, sexo feminino, branca, brasileira, dona de casa, irregularmente ativa através da análise pelo
International Physical Activity Questionnaire (IPAQ) versão longa, índice de massa corporal de $20 \mathrm{~kg} / \mathrm{m}^{2}$, diagnósticada com Insuficiência Cardíaca Crônica (ICC), dislipidemia e Hipertenssão Arterial Sistêmica (HAS), todas há 20 anos. Não realizou intervenção com exercício anteriormente, somente tratamento farmacológico. Ingressou no serviço de Reabilitação Cardiovascular e Metabólica (RCVM) na clínica ACTUS CORDIOS em agosto de 2015, com diagnóstico de DM2 há 15 anos, com glicemia de jejum de 346mg/ dL e Hemoglobina Glicada (HbA1c) de 12\%. Em uso de insulina (NPH e ultrarrápida) há 6 anos, Losartana (50mg) e Atorvastatina (20mg). Como a paciente referiu cansaço aos esforços leves, como fazer compras ou subir um lance de escada, a ICC foi classificada funcionalmente como grau III, pelo especialista responsável. Sua principal queixa além do cansaço, era o uso diário da insulina subcutânea. No histórico familiar foram encontrados fatores como dislipidemia, HAS e DM2.

Ao exame físico foi identificada a sarcopenia, determinada pela circunferência da panturrilha (valor avaliado de $28 \mathrm{~cm}$; valor de referência de $33 \mathrm{~cm})^{6}$. A pressão arterial sistêmica (PAS) foi mensurada com estetoscópio Littmann Classic III e esfigmomanômetro da marca Welch Allyn, modelo Durashock Ds44br, obtendo o valor $185 \times 95 \mathrm{mmHg}$. A força muscular avaliada pela escala Medical Research Council (MRC) foi de grau 4 e no Teste de Caminhada de 6 Minutos (TC6) foi obtido um valor de $300 \mathrm{~m}$, abaixo do previsto para idade $(502,5 \mathrm{~m})$, pela equação de Iwama et al. ${ }^{7}$.

\section{Estratégia de prescrição}

Para avaliação da intensidade do exercício físico, foi utilizada a escala de percepção subjetiva de Borg (com medidas que variam de 6 a 20 pontos) $)^{8}$. Antes de cada sessão, a voluntária recebeu as instruções sobre o funcionamento do instrumento e o objetivo de relatar a sensação de fadiga cardiorrespiratória. Os valores de PAS, frequência cardíaca (FC) e glicemia capilar foram mensuradas respectivamente através do estetoscópio Littmann Classic III 5620 e Esfigmomanômetro Aneróide Welch Allyn, modelo Durashock Ds44-br, oxímetro de pulso G-tech portátil monitor de dedo oled e o Medidor de Glicemia One Touch Ultra Plus Flex, no início, durante e no final de cada sessão.

O programa aplicado ao estudo consistiu em exercícios resistidos e cíclicos, realizados duas vezes por semana, com cerca de 80 minutos de duração em cada sessão, durante o período de quatro meses. 
No primeiro mês, foi realizado somente treino adaptativo neuromuscular com duas séries de 15 repetições, com intervalos de 2 minutos, sendo dois exercícios para membros superiores (MMSS): Rosca direta para bíceps e elevação para ombro e, dois para membros inferiores (MMSS): Marcha estática com tornozeleira e exercício para panturrilha, (com intensidade entre 9 a 11 da escala de Borg) e esteira ergométrica por 10 minutos em intensidade leve (Borg de 9 a 11).

A partir do segundo mês, as sessões foram realizadas com o intuito de aumentar a massa muscular focando, portanto, nos exercícios neuromusculares com duas séries de 8 repetições com intervalo de dois minutos entre as séries para 4 exercícios multiarticulares divididos em: supino com altere e remada para MMSS e agachamento e marcha estática com tornozeleira para MMII, realizados em dias alternados. Intensidade determinada para um Borg de 12 a 14.

A partir do segundo mês o exercício cíclico na esteira ergométrica foi modificado sendo, por 5 minutos em intensidade leve (Borg de 9 a 11) mais 10 minutos em intensidade moderada (Borg de 12 a 14), finalizando com 3 minutos de desaquecimento em intensidade regressiva. Ao final de cada sessão foi realizado técnicas de relaxamento com massoterapia. O programa teve frequência semanal de 2 dias. As cargas do exercício neuromuscular e a velocidade da esteira foram ajustadas progressivamente nos demais meses de intervenção, seguindo o prescrito no programa de acordo com a escala de Borg.

O treinamento muscular inspiratório (TMI) começou a ser realizado a partir do terceiro mês, 30 repetições, cinco vezes na semana, na clínica, em carga a 30\% da PImáx avaliada.

\section{Resultados}

Após 16 semanas de RCVM houve redução de 70\% na glicemia de jejum e de $37,5 \%$ na HbA1c, demonstrando controle dos níveis glicêmicos inclusive pós-prandiais. Concomitante observamos $128 \%$ de melhora da capacidade funcional avaliada através do TC6, como apresentado na Tabela 1.

Tabela 1. Comparação das variáveis antes e após 16 semanas de intervenção através de um programa de reabilitação cardiovascular e metabólica para DM2

\begin{tabular}{|c|c|c|c|c|}
\hline Variáveis & Pré-RCVM & Pós-RCVM & $\%$ de Melhora & $\begin{array}{c}\text { Valor de } \\
\text { normalidade }\end{array}$ \\
\hline $\mathrm{HbA1c}(\%)$ & 12 & 7,5 & 37,5 & Até $5,6^{\#}$ \\
\hline Glicemia de jejum (mg/dL) & 346 & 105 & 70 & $<100^{\#}$ \\
\hline Colesterol total (mg/dL) & 158 & 108 & 32 & $<200 *$ \\
\hline $\mathrm{LDL}(\mathrm{mg} / \mathrm{dL})$ & 95 & 58 & 61 & $<130 *$ \\
\hline $\mathrm{HDL}(\mathrm{mg} / \mathrm{dL})$ & 31 & 41 & 32 & $>60 *$ \\
\hline Triglicerídeos (mg/dL) & 115 & 97 & 16 & $\leq 150 *$ \\
\hline PAS (mmHg) & 185 & 139 & 25 & $<140 *$ \\
\hline $\mathrm{PAD}(\mathrm{mmHg})$ & 95 & 85 & 5 & $<90 *$ \\
\hline TC6 (metros) & 350 & 800 & 128 & $502,5^{\$}$ \\
\hline
\end{tabular}

HbA1c - Hemoglobina glicada; HDL - Lipoproteína de alta densidade; LDL - Lipoproteína de baixa densidade; PAD Pressão Arterial Diastólica; PAS - Pressão Arterial Sistólica; RCVM - Reabilitação Cardiovascular e Metabólica; TC6 - teste de caminhada de 6 minutos. \#Segundo a Diretriz da Associação Americana de Diabetes"; *Segundo a atualização da Diretriz Brasileira de Dislipidemia e Aterosclerose-2017 ${ }^{10}$ Segundo a equação de Iwama et al., ${ }^{7}$ 
O programa de RCVM também propiciou a redução de fármacos administrados para controle da dislipidemia e HAS, bem como foi retirada a utilização da insulina subcutânea por orientação do médico endocrinologista, como apresentado na Tabela 2.

Tabela 2. Fármacos utilizados antes e após o programa de reabilitação cardiovascular e metabólica para DM2

\begin{tabular}{cll}
\hline Fármacos & Pré-RCVM & Pós-RCVM \\
\hline Atorvastatina & $1 \times$ por dia (80mg) & 1x por dia (10mg) \\
\hline Monohidrato de Isossorbida & $2 \times$ por dia & $1 \times$ por dia \\
\hline Insulina Lantus (ação lenta - 24h) & $1 \times$ por dia & Removida \\
\hline Insulina Apidra (ultrarrápida) & $3 \times$ por dia & Removida \\
\hline
\end{tabular}

RCVM - Reabilitação Cardiovascular e Metabólica.

\section{Discussão}

Este relato de caso demonstrou que um programa de RCVM supervisionado foi capaz de melhorar o controle glicêmico levando a redução da quantidade de fármacos, aumento da capacidade funcional e meIhora do perfil lipídico e redução da PAS em uma paciente com DM e ICC classe III.

O início da utilização do aparelho para o treinamento muscular inspiratório ocorreu de forma tardia em função do atraso na entrega do equipamento, mesmo assim, os resultados foram satisfatórios e levantam lacunas sobre o quão extensos podem ser os benefícios do exercício físico quando feitos de forma personalizada para cada paciente. Ainda neste sentido, a aplicação do TMI é um grande aliado na reabilitação por atuar diretamente sobre o metaborreflexo ${ }^{11}$, portanto, acreditamos que a inserção dessa estratégia logo no início do programa poderia ter fornecido resultados ainda melhores no condicionamento da paciente.

Estudos clínicos de causa e efeito têm apontado que o exercício físico é um importante recurso terapêutico para controle glicêmico em diabéticos ${ }^{12,13}$. Segundo o estudo de Chibalin et al. ${ }^{12}$ o exercício aumenta a expressão e ação dos receptores de insulina (IRS-1 e IRS-2) presentes na membrana plasmática das células musculares estriadas (esquelética e cardíaca). A insulina é o hormônio responsável por iniciar a sinalização da translocação intracelular de proteínas transportadoras de glicose (GLUTs) para o sarcolema. O GLUT-4, presente nas células musculares estriadas, é o responsável pelo processo de difusão facilitada da glicose sanguínea para o interior das células musculares e sua produção também é induzida pelo exercício ${ }^{12,14}$. Portanto, o aumento na expressão e atividade dos receptores de insulina, presentes no sarcolema, e o aumento da expressão intracelular do GLUT-4, ambos promovidos de forma crônica pelo exercício físico, contribuem fortemente para melhorar o metabolismo glicêmico em pacientes com diabetes mellitus ${ }^{15}$.

No estudo de Andrade et al. ${ }^{4}$ foi verificado que um programa de treinamento aeróbico cíclico na esteira de 12 semanas promoveu efeitos significativos na redução dos níveis glicêmicos pós prandial e hemoglobina glicada em pacientes com DM2. Contudo, esses efeitos foram visíveis a longo prazo, a partir da $8^{a} \mathrm{e}$ $12^{a}$ semana, evidenciando que os benefícios oriundos dos exercícios físicos são adquiridos de maneira substancial após 2 ou 3 meses, reforçando a importância de programas de treinamentos regulares para a prevenção e tratamento da DM2.

No entanto, o efeito do exercício físico vai além do equilíbrio da ação insulínica, já que, a translocação do GLUT-4, ocorre também pela liberação de cálcio do retículo sarcoplasmático durante a contração muscular, mecanismo este, não dependente da insulina e que perdura por até duas horas pós-exercício. Existem ainda, evidências nos estudos de $\operatorname{Lima}^{16}$ e Junior et al. ${ }^{17}$ de que o exercício físico estimula a produção de substâncias autócrinas e parácrinas, como a calicreína, adenosina e o óxido nítrico que também promovem a sinalização para a translocação do GLUT-4. Desta 
forma, o efeito do exercício não está restrito somente aos pacientes que possuem produção de insulina normal (como a maioria dos pacientes com DM2), mas, também aos que tem produção diminuída ou ausente, como no diabetes mellitus tipo $\mathbf{I}^{17}$.

Aprofundando essa discussão sobre os mecanismos não dependentes de insulina, lembramos que em repouso parte do GLUT-4 permanece armazenado em vesículas e sua translocação por meio do exercício tem efeito mais prolongado por aumentar a taxa de exocitose e atenuar a endocitose desta proteína. As sinalizações podem ocorrer via fosforilação da proteína quinase ativada por monofosfato de adenosina (AMPK), que é estimulada pela quinase B1 do fígado (LKB1), expressa com a mudança do balanço celular entre adenosina monofosfato (AMP) e adenosina trifosfato (ATP), aumento e redução respectivamente, durante o exercício. A partir da ativação da AMPK alguns eixos podem determinar o transporte do GLUT-4 à periferia, como a diminuição da atividade de proteínas que impedem a liberação vesicular do GLUT-4 (mediada pelo Fator estimulador de GLUT-4), ativação da proteína AS160 que sinaliza a proteína Rab-GTP para início do transporte. Outra função da AMPK está ligada ao aumento da expressão gênica de GLUT-4, com consequente aumento na quantidade de proteínas transportadoras disponíveis ${ }^{18}$.

Importante ressaltar que um programa de RCVM supervisionada contribui não somente pelos mecanismos biológicos promovidos pelo exercício físico, mas também pelos fatores pleiotrópicos, como diminuição da ansiedade e estresse emocional e melhor controle dietético o que, por consequência, influenciam nos resultados da RCVM ${ }^{19}$. Finalmente, a soma desses mecanismos (biológicos e pleiotrópicos), pode reduzir ou até abolir a utilização de fármacos por muitos dos pacientes, como foi observado neste relato, o que implica diretamente na relação custo-benefício da RCVM e na melhora da qualidade de vida observada em pacientes inseridos nesses programas.

\section{Conclusão}

Os resultados descritos neste relato de caso evidenciam que um programa reabilitação cardiovascular e metabólica supervisionado é capaz de modificar os níveis glicêmicos, dispensando o uso de insulina subcutânea e promovendo melhora da capacidade funcional, como visto nessa paciente com insuficiência cardíaca crônica grau III e diabetes mellitus tipo 2 .

\section{Contribuições dos autores}

Cerqueira DGLES, Oliveira IM, Petto J conceberam o estudo e desenho da pesquisa. Cerqueira DGLES, Oliveira IM, Jesus TC, Petto J. analisaram e interpretaram os dados. Cerqueira DGLES, Sacramento MS, Santos VR, Jesus TC, Petto J redigiram o manuscrito. Sacramento MS, Petto J fizeram a revisão crítica do manuscrito quanto ao conteúdo intelectual importante.

\section{Conflitos de interesses}

Dr. Petto, Dr. Cerqueira e Dr. Sacramento informam recebimento de honorários da ACTUS CORDIOS, sem vínculos com este relato. Nenhum conflito financeiro, legal ou político envolvendo terceiros (governo, empresas e fundações privadas, etc.) foi declarado para nenhum aspecto do trabalho submetido (incluindo, mas não se limitando a subvenções e financiamentos, participação em conselho consultivo, desenho de estudo, preparação de manuscrito, análise estatística, etc.) pelos demais autores.

\section{Referências}

1. Guariguata L, Whiting DR, Hambleton I, Beagley J, Linnenkamp U, Shaw JE. Global estimates of diabetes prevalence for 2013 and projections for 2035. Diabetes Res Clin Pract. 2014;103(2):137-49. doi: 10.1016/j.diabres.2013.11.002

2. Schmidt MI, Duncan BB, Mill JG, Lotufo PA, Chor D, Barreto SM et al. Perfil da Coorte: Estudo Longitudinal da Saúde do Adulto (ELSA-Brasil). International Journal of Epidemiology. 2015;44(1):6875. doi: $10.1093 /$ ije/dyu027

3. Arsa G, Lima L, Almeida SS, Moreira SR, Campbell CSG, Simões HG. Diabetes Mellitus tipo 2: Aspectos fisiológicos, genéticos e formas de exercício físico para seu controle. Rev Bras Cineantropom Desempenho Hum. 2009;11(1):103-11. doi: 10.5007/1980-0037.2009v11n1p103

\footnotetext{
4. Andrade EA, Fett CA, Vieira Junior RC, Voltarelli FA. Exercício físico de moderada intensidade contribui para o controle de parâmetros glicêmicos e clearance de creatina em pessoas com Diabetes Mellitus tipo 2. Rev Bras Cienc e Mov. 2016;24(1):118126. doi: $10.18511 /$ rbcm.v24i1.5975
}

5. Sociedade Brasileira de Diabetes. Diretrizes da Sociedade Brasileira de Diabetes. 2017-2018. [Internet]. 2019. Disponível em: https://www.diabetes.org.br/profissionais/images/2017/diretrizes/ diretrizes-sbd-2017-2018.pdf 
6. Pagotto V, Santos KF, Malaquias SG, Bachion MM, Silveira EA. Circunferência da panturrilha: validação clínica para avaliação de massa muscular em idosos. Rev Bras Enferm. 2018;71(2):322-328. doi: $10.1590 / 0034-7167-2017-0121$

7. Iwama AM, Andrade GN, Shima P, Tanni SE, Godoy I, Dourado VZ. The six minute walk test and body weight-walk distance product in healthy Brazilian subjects. Braz J Med Biol Res. 2009;42(11):1080-5. doi: 10.1590/S0100-879X2009005000032

8. Cabral LL, Lopes PB, Wolf R, Stefanello JMF, Pereira G. A systematic review of cross-cultural adaptation and validation of Borg's Rating Of Perceived Exertion Scale. J Phys Educ. 2017;28(1). doi: 10.4025/jphyseduc.v28i1.2853

9. American Diabetes Association. Classification and Diagnosis of Diabetes: Standards of Medical Care in Diabetes - 2019. Diabetes Care. 2019;42(1):13-28. doi: 10.2337/dc19-S002

10. Faludi AA, Izar MCO, Saraiva JFK, Chacra APM, Bianco HT, Afiune Neto A et al. Atualização da Diretriz Brasileira de Dislipidemias e Prevenção da Aterosclerose - 2017. Arq Bras Cardiol 2017;109(2 supl 1):1-76. doi: 10.5935/abc.20170121

11. Lin SJ, McElfresh J, Hall B, Bloom R, Farrell K. Inspiratory muscle training in patients with heart failure: a systematic review. Cardiopulm Phys Ther J. 2012;23(3):29-36.

12. Chibalin AV, Yu M, Ryder JW, Song XM, Galuska D, Krook A et al. Exercise-induced changes in expression and activity of proteins involved in insulin signal transduction in skeletal muscle: Differential effects on insulin-receptor substrates 1 and 2. Proc Natl Acad Sci U S A. 2000;97(1):38-43. doi: 10.1073/pnas.97.1.38

13. Pessin JE, Saltiel AR. Signaling pathways in insulin action: molecular targets of insulin resistance. Journal of Clinical Investigation. 2000;106(2):165-9. doi: 10.1172/jci10582

14. White MF. O sistema de sinalização do IRS: Uma rede de proteínas de acoplamento que medeiam a ação da insulina. Mohcuklrand Cellular Biochemistn. 1998;182:3-11. doi:

10.1007/978-1-4615-5647-3_1

15. Camporez JPG, Almeida FN, Marçal AC. Efeitos do exercício físico sobre a via de sinalização da insulina. Revista Mackenzie de Educação Física e Esporte. 2013;12(2): 172-186.

16. Lima MF. Análise dos efeitos do exercício fisíco aplicado aos pacientes com hipertensão arterial sistêmica e diabetes de mellitus assistidos pela equipe de estratégia saúde e familia da cidade de Paracatu-MG. Humanidades e tecnologia (FINOM). 2019;1(16):474-493.

17. Souza Junior TP, Asano RY, Prestes J, Sales MPM, Coelho JMO, Simões HG. Óxido nítrico e exercício: Uma revisão nitric oxide and exercise: a short review. Rev Educ Fis. 2012;23(3):469-481. doi: 10.4025/reveducfis.v23i3.11738
18. Pereira RM, Moura LP, Muñoz VR, Silva ASR, Gaspar RS, Ropelle ER et al. Molecular mechanisms of glucose uptake in skeletal muscle at rest and in response to exercise. Motriz: Rev Educ Fis. 2017;23(Special Issue):e101609. doi: 10.1590/s19806574201700 si0004

19. Batista Jl, Oliveira A. Efeitos psicofisiológicos do exercício físico em pacientes com transtornos de ansiedade e depressão. Corpoconsciência. 2016;19(3):1-10. 